\title{
Detection and measurement of expanding ionized shells around young star clusters
}

\author{
John Beckman ${ }^{1,2,3}$ Artemi Camps Fariña $a^{1,2}$, Javier Zaragoza \\ Cardiel $^{1,2,4}$, Joan Font ${ }^{1,2}$ \\ ${ }^{1}$ Instituto de Astrofísica de Canarias, La Laguna, Spain ; email: jeb@iac.es \\ ${ }^{2}$ Departamento de Astrofísica, Universidad de la Laguna, Spain \\ ${ }^{3}$ Consejo Superior de Investigaciones Científicas, Spain \\ ${ }^{4}$ Instituto de Astronomía, UNAM, México D.F.
}

\begin{abstract}
We present a new method (BUBBLY)for detecting expanding components of ionized gas using integral field spectroscopy, showing its capabilities not only in detecting but also in obtaining the key physical parameters of the expanding shells: their expansion velocities and masses. The main advantages are that the detection is performed automatically via software and that we can derive most parameters of the shells, so it is suitable for detailed studies of feedback in nearby galaxies. The software can easily be configured to run on any data cube mapping an emission line over a spatial field. We also present results obtained by running BUBBLY on observations of $\mathrm{H} \alpha$ emission with the Fabry-Perot spectrograph $\mathrm{GH} \alpha \mathrm{FaS}$ : two sets of spectacular results at widely different spatial scales: the Antennae galaxies show multiple giant bubbles of size $\sim 300$ pc around the brightest clusters, while inside a region in M33 we find three nested supernova remnants, with which we can study the feedback on the molecular gas surrounding the cluster.
\end{abstract}

Keywords. ISM: jets and outflows, supernova remnants, galaxies: star clusters

\section{Introduction and method}

Massive stars in clusters produce expanding shells injecting energy into the surrounding ISM via winds and supernovae. They are a key to feedback processes in galaxy evolution. Stellar feedback is a leading candidate to solve astrophysical problems, such as metal distribution throughout the disc (Spitoni et al.(2009)), long-term star formation through interaction with the galactic corona (Marasco et al.(2012)) and even the dissipation of dark matter cusps (Pontzen \& Governato(2012)). We have developed a sensitive method (Camps-Fariña et al.(2015)) to detect these shells using Fabry-Pérot spectroscopy, and tested it with the Galaxy $\mathrm{H} \alpha$ FabryPerot System $(\mathrm{GH} \alpha \mathrm{FaS})$,on the William Herschel Telescope at La Palma Observatory. GH $\alpha \mathrm{FaS}$ allows us to measure $\mathrm{H} \alpha$ emission over a 3.4'x3.4' field with seeing-limited resolution and velocity resolution of $\sim 6 \mathrm{~km} \mathrm{~s}^{-1}$.

\section{Results}

We present results from three objects, a strongly interacting pair of galaxies: the Antennae, a more quiescent grand-design spiral M83 and lastly a moderately-sized region in the nearby galaxy M33.

In the Antennae we found large scale bubbles of radii on the order of 300-400 pc, with high velocities for objects of this size, ranging from $\sim 60-115 \mathrm{~km} / \mathrm{s}$. These bubbles are tightly correlated with the largest OB associations, whose stellar winds and supernova explosions should be enough to power them. Especially interesting is the bubble associated with the brightest HII region, which is the clearest example in the galaxy, showing distinct projection effects, as well as the fastest and most energetic, with $(49 \pm 14) \cdot 10^{51} \mathrm{erg}$ (The next most energetic bubble has $\left.(15 \pm 5) \cdot 10^{51} \mathrm{erg}\right)$. 


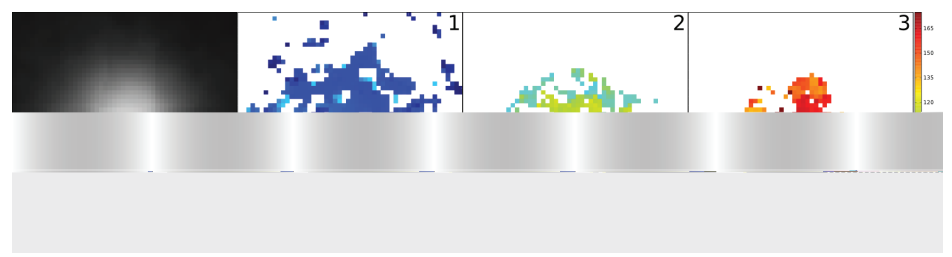

Figure 1. Expansion maps of the three detected bubbles in M33. On the left, for comparison, is the total surface brightness in $\mathrm{H} \alpha$, while the next three images show the detected expansion velocity on each pixel, all on the same velocity scale. They are roughly concentric and their centres coincide with that of the region itself.

Table 1. Physical properties of the triple bubble in M33

\begin{tabular}{llllll} 
& Radius $(\mathrm{pc})$ & $\mathrm{v}_{\text {exp }}(\mathrm{km} / \mathrm{s})$ & Mass $\left(\mathrm{M}_{\odot}\right)$ & $\mathrm{E}_{k}\left(10^{49}\right.$ erg $)$ & Age $(\mathrm{kyr})$ \\
Bubble 1 & $22 \pm 2$ & $55 \pm 5$ & $337 \pm 34$ & $1.0 \pm 0.2$ & $114 \pm 16$ \\
Bubble 2 & $16 \pm 2$ & $115 \pm 12$ & $118 \pm 12$ & $1.6 \pm 0.3$ & $40 \pm 6$ \\
Bubble 3 & $12.5 \pm 1$ & $165 \pm 17$ & $54 \pm 5$ & $1.4 \pm 0.3$ & $21 \pm 3$ \\
Notes: & \multicolumn{3}{c}{}
\end{tabular}

The quantities have been calculated with a lower limit for shell thickness, this implies that mass and kinetic energy are lower limits. Even so, the dependence on shell thickness is $\sqrt{\Delta R}$, so a factor 10 increase in the thickness would increase mass and energy a factor $\sim 3$.

M83 is a more quiescent galaxy, which is clear also from the expansion map. We find smaller $(100 \mathrm{pc})$, far less energetic $1-2 \cdot 10^{51}$ erg bubbles expanding at velocities around $100 \mathrm{~km} / \mathrm{s}$. The exception is the nucleus of the galaxy, where we observe many secondary components in velocity overlapping along the line of sight, suggesting a violent environment with intense star formation and feedback. The bubbles are more uniform than those of the Antennae, suggesting a recent galaxy-wide burst of star formation.

\subsection{Triple bubble in $M 33$}

M33 is a very nearby galaxy, so we can observe in much more detail. In a field on the southern arm containing several moderately sized (20-60 pc) HII regions, we found one which shows three sets of coherent expanding components, implying three concentric expanding shells(see Fig 1). We used emission line ratio diagnostics to show that they are supernova remnants, which must come from stars with similar masses. A key physical parameter of the bubbles (see Table 1) in this case is their mass. As the supernovae must have exploded almost simultaneously on astrophysical time-scales, the first shell should have swept-up practically all the surrounding material, leaving almost nothing for the following two to sweep up, but their masses are far too big for this, suggesting that the medium inside the HII region is very clumpy, with embedded cold clumps surviving since the cluster birth. These would not be fully destroyed by the first passing shock, and could interact with the later shells providing the necessary mass-loading. Including the effect of cold clumps on the expanding supernova remnants makes the physical parameters fit better, as for a homogeneous medium the remnants would be faster, and with more energy for their size, than measured.

\section{References}

Spitoni, E., Matteucci, F., Recchi, S., Cescutti, G., \& Pipino, A. 2009, A\&A, 504, 87

Marasco, A., Fraternali, F., \& Binney, J. J. 2012, MNRAS, 419, 1107

Pontzen, A., \& Governato, F. 2012, MNRAS, 421, 3464

Camps-Fariña, A., Zaragoza-Cardiel, J., Beckman, J. E., et al. 2015, MNRAS, 447, 3840 